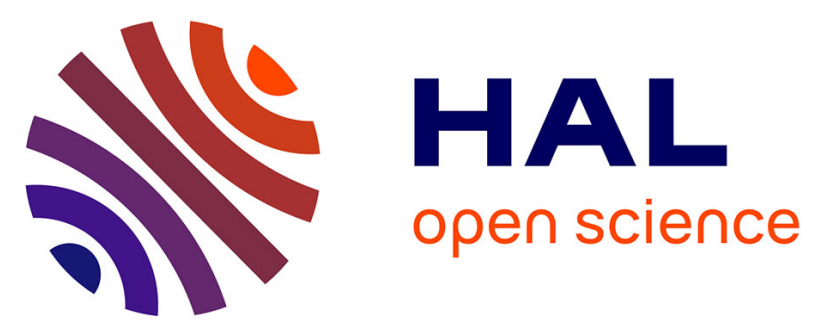

\title{
Robust Augmented Reality registration method for Localization of Solid Organs' Tumors Using CT-derived Virtual Biomechanical Model and Fluorescent Fiducials
}

Seong-Ho Kong, Nazim Haouchine, Renato Soares, Andrey S Klymchenko, Bohdan Andreiuk, Bruno Marques, Galyna Shabat, Thierry Piéchaud, Michele Diana, Stéphane Cotin, et al.

\section{To cite this version:}

Seong-Ho Kong, Nazim Haouchine, Renato Soares, Andrey S Klymchenko, Bohdan Andreiuk, et al.. Robust Augmented Reality registration method for Localization of Solid Organs' Tumors Using CT-derived Virtual Biomechanical Model and Fluorescent Fiducials. Surgical Endoscopy, 2016, 10.1007/s00464-016-5297-8 . hal-01314963v2

\author{
HAL Id: hal-01314963 \\ https://hal.science/hal-01314963v2
}

Submitted on 17 Nov 2016

HAL is a multi-disciplinary open access archive for the deposit and dissemination of scientific research documents, whether they are published or not. The documents may come from teaching and research institutions in France or abroad, or from public or private research centers.
L'archive ouverte pluridisciplinaire HAL, est destinée au dépôt et à la diffusion de documents scientifiques de niveau recherche, publiés ou non, émanant des établissements d'enseignement et de recherche français ou étrangers, des laboratoires publics ou privés. 


\title{
Robust augmented reality registration method for localization of solid organs' tumors using CT-derived virtual biomechanical model and fluorescent fiducials
}

\author{
Seong-Ho Kong ${ }^{1,2} \cdot$ Nazim Haouchine ${ }^{3} \cdot$ Renato Soares $^{1} \cdot$ Andrey Klymchenko $^{4}$ • \\ Bohdan Andreiuk ${ }^{4} \cdot$ Bruno Marques $^{3} \cdot$ Galyna Shabat $^{5} \cdot$ Thierry Piechaud $^{6}$.

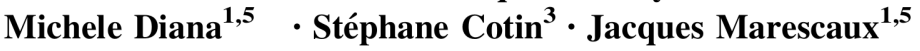

\begin{abstract}
Background Augmented reality (AR) is the fusion of computer-generated and real-time images. AR can be used in surgery as a navigation tool, by creating a patientspecific virtual model through 3D software manipulation of DICOM imaging (e.g., CT scan). The virtual model can be superimposed to real-time images enabling transparency visualization of internal anatomy and accurate localization of tumors. However, the 3D model is rigid and does not take into account inner structures' deformations. We present a concept of automated AR registration, while the organs undergo deformation during surgical manipulation, based on finite element modeling (FEM) coupled with optical imaging of fluorescent surface fiducials.
\end{abstract}

Presented at the SAGES 2016 Annual Meeting, March 16-19, 2016, Boston, MA.

Michele Diana

michele.diana@ircad.fr; michele.diana@ihu-strasbourg.eu

1 IHU-Strasbourg, Institute of Image-Guided Surgery, Strasbourg, France

2 Department of Surgery, Seoul National University Hospital, Seoul, Korea

3 Institut national de recherche en informatique et en automatique (INRIA) Mimesis, Strasbourg, France

4 Biophotonic and Pharmacology Lab, UMR 7213 CNRS, Pharmacological Faculty, University of Strasbourg, Strasbourg, France

5 IRCAD, Research Institute against Cancer of the Digestive System, 1, Place de l'Hôpital, 67091 Strasbourg, France

6 Division of Urology, Clinique Saint-Augustin, Bordeaux, France
Methods Two $10 \times 1 \mathrm{~mm}$ wires (pseudo-tumors) and six $10 \times 0.9 \mathrm{~mm}$ fluorescent fiducials were placed in ex vivo porcine kidneys $(n=10)$. Biomechanical FEM-based models were generated from CT scan. Kidneys were deformed and the shape changes were identified by tracking the fiducials, using a near-infrared optical system. The changes were registered automatically with the virtual model, which was deformed accordingly. Accuracy of prediction of pseudo-tumors' location was evaluated with a CT scan in the deformed status (ground truth). In vivo: fluorescent fiducials were inserted under ultrasound guidance in the kidney of one pig, followed by a CT scan. The FEM-based virtual model was superimposed on laparoscopic images by automatic registration of the fiducials.

Results Biomechanical models were successfully generated and accurately superimposed on optical images. The mean measured distance between the estimated tumor by biomechanical propagation and the scanned tumor (ground truth) was $0.84 \pm 0.42 \mathrm{~mm}$. All fiducials were successfully placed in in vivo kidney and well visualized in near-infrared mode enabling accurate automatic registration of the virtual model on the laparoscopic images.

Conclusions Our preliminary experiments showed the potential of a biomechanical model with fluorescent fiducials to propagate the deformation of solid organs' surface to their inner structures including tumors with good accuracy and automatized robust tracking.

Keywords Augmented reality - Automatic registration · Optical imaging · Finite element modeling · Solid organ tumor · Fluorescence-guided surgery $\cdot$ Fiducials

Along with increases in early detection of the tumors and interests in the quality of life after surgery, individualized 
tailored resection methods have been replacing the traditional radical wide resection in diverse organs $[1,2]$. Accurate tumor localization is crucial to ensure both radicality and organ function preservation in tailored resection. It is frequently challenging to accurately localize and determine the margin of the tumors in solid organs during minimally invasive surgeries, especially when the tumor is located deep inside organs or when they are only partially exposed [3, 4].

Augmented reality (AR) technologies, which generate patient-specific virtual model through 3D software manipulation of DICOM imaging (e.g., CT or MRI) and provide superimposed images in the operation field to obtain the enhanced real-time localization, are receiving increasing interest as effective and useful tools for the accurate tailored operation [5-10].

The inability to simulate the deformation of the organ during surgery, and the requirement of frequent manual re-localization to synchronize the virtual model and the laparoscopic operative images are important limitations of the conventional rigid virtual model. To overcome these limitations, we have developed a real-time biomechanical model based on a finite element modeling (FEM) method. FEM allows to simulate soft tissue behavior, taking into account mechanical properties of parenchyma, internal tubular structures, and tumors. This biomechanical model is expected to predict inner deformations by propagation of the observed surface changes [11].

We have opted for a surface fiducials-based tracking method for robust automatic re-localization. The gold fiducials, characterized by small size, visibility at imaging modalities such as CT, and minimal tissue reaction, are safely used for localization of tumors in stereotactic radio surgery (Cyberknife ${ }^{\circledR}$ ) treatment of malignancies in various organs such as the lung, liver, prostate, and metastatic lymph nodes [12-14]. We hypothesized that the fiducials on the surface of the solid organ can be used as the landmark for a robust re-localization as well as for a computed re-modeling of the virtual model in a deformed shape. We also used near-infrared fluorescent coating on the fiducials to increase the visibility and selectivity, because near-infrared fluorescent imaging has been known to have the advantage of deep penetration and high signal/background ratio [15].

The purpose of this study was to validate whether the real-time tracking method, combining biomechanical virtual models and the fluorescent dye-coated surface fiducials, is effective for enhanced robustness through automatic registration and for the accuracy of tumor localization in deformed solid organs, using ex vivo and in vivo porcine kidney models.

\section{Materials and methods}

\section{Fluorescent gold fiducials}

Commercially available gold fiducials (Flexicoil $^{\mathrm{TM}}$, CIVCO, IOWA, USA), which are made of biocompatible pure gold (99.99\% minimum), measure $10 \mathrm{~mm}$ in length and $0.9 \mathrm{~mm}$ in diameter, were used. The fiducials have a helix shape to minimize the likelihood of migration and artifacts. Fiducials were coated with a custom-made, biocompatible, near-infrared fluorescent polymeric material (Fig. 1).

\section{Ex vivo experiments}

Ex vivo experiments were planned to examine the robustness of the re-localization and accuracy of the prediction of the location of the tumor in deformed kidneys.

A total of ten ex vivo kidneys (mean size $100.2 \pm 9.8 \mathrm{~mm}$ ) were harvested from pigs (Sus scrofa domesticus, ssp Large White), which were involved in educational courses in minimally invasive surgery. The kidneys were immediately stored in the deep freezer $\left(-20^{\circ} \mathrm{C}\right)$ and slowly thawed in the warm water bath during a few hours on the days of the experiments. The kidney parenchyma was filled with saline injected through the artery, and both the renal artery and veins were ligated.

Two $10 \times 1 \mathrm{~mm}$ sized metal wires were used to simulate tumors (pseudo-tumors) and were inserted into the parenchyma of anterior and posterior side of the kidney using a 18-gauge placement needle (CIVCO, IOWA, USA), either in the upper half or in lower half of the kidney, which were randomly selected. Six fiducials were widely placed using the same placement needle around the expected area of the pseudo-tumor, with a depth that exposes 1-2 $\mathrm{mm}$ of the tip outside the kidney. CT was performed for a generation of the virtual model of the kidney including the pseudo-tumor.

The kidney was observed with the near-infrared fluorescent camera (D-Light P, Karl Storz, Tuttlingen; Germany). A deformation was applied to the upper or lower part of the kidney, including the pseudo-tumors, and the kidney in the deformed status was left recumbent over a small wedge. Then a second CT scan was performed to image the deformed kidney.

The changes in the fiducials' location were detected by laparoscopic view, and new locations were applied to the virtual model to automatically generate the deformation. The accuracy of the prediction of displacement of the pseudo-tumors was examined by means of comparing the virtual model and the ground truth information obtained by the second CT scan. 


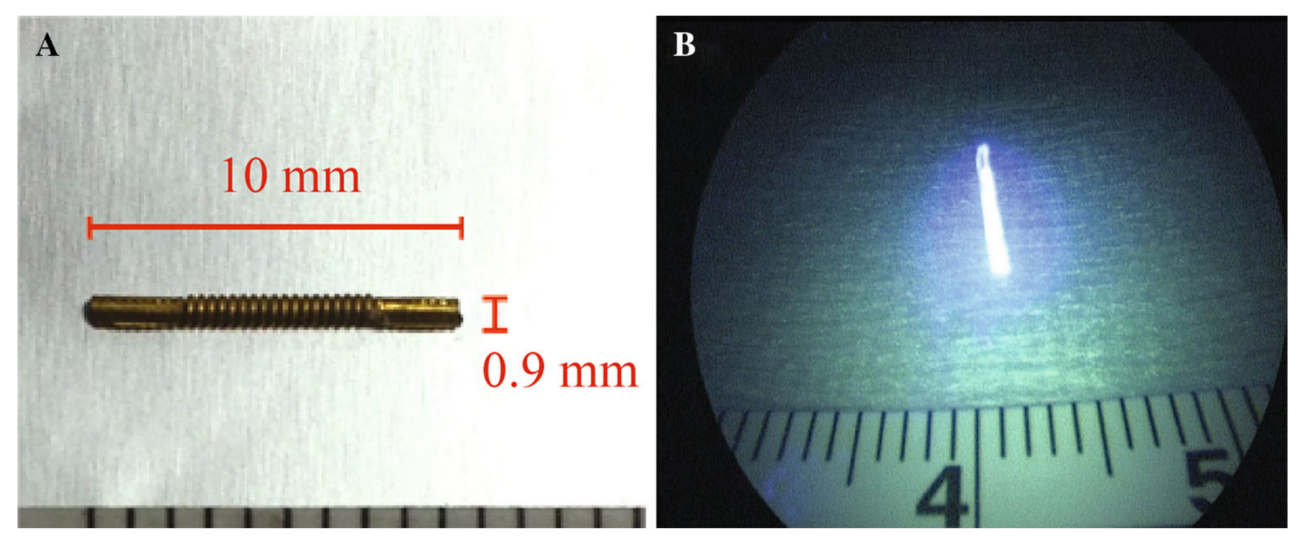

Fig. 1 Surface fiducials. A White light view, B near-infrared mode of laparoscopic camera

\section{In vivo experiments}

In vivo tests to validate the process were performed in a $25-30-\mathrm{kg}$ pig. In accordance with the ethical principle of reduction, the animal was included in the present study at the end of a different experimental protocol (No. 38.2013.01.048, acronym Penumbra), which has received full approval by the Ethical Committee on animal experimentation, and is still ongoing at our institute. All animals were managed according to the directives of the European Community Council (2010/63/EU). Pigs were fasted for $24 \mathrm{~h}$ before surgery with free access to water. Premedication by intramuscular injection of ketamine $(20 \mathrm{mg} / \mathrm{kg})$ and azaperone $(2 \mathrm{mg} / \mathrm{kg})$ (Stresnil; Janssen Cilag, Belgium) was administered 10 min before surgery. Induction was achieved with intravenous propofol $(3 \mathrm{mg} /$ $\mathrm{kg})$ combined with rocuronium $(0.8 \mathrm{mg} / \mathrm{kg})$. Anesthesia was maintained with $2 \%$ isoflurane. At the end of the procedure, the animal was humanely sacrificed with an intravenous injection of a lethal dose of potassium chloride.

Six fluorescent-coated fiducials were widely placed on the anterior surface of the kidney via 18 -gauge placement needle guided, by percutaneous approach under ultrasonography guidance. A CT scan was then performed to generate $3 \mathrm{D}$ virtual model.

A laparoscopic near-infrared camera (D-Light P, Karl Storz; Tuttlingen, Germany) was inserted into the 12-mm trocar placed in the supra-umbilical area, and the kidney and the fiducials were observed and recorded at white light mode and at near-infrared fluorescent mode.

The location of fiducials detected by optical imaging was used to re-localize the virtual 3D model over the laparoscopic view.

The accuracy of the re-localization was estimated by examining the similarity of the boundary of the kidney between the virtual model and the laparoscopic image using Dice's coefficient, as previously described [16].

\section{Augmented reality using image-driven biomechanical models}

The key idea of our method is to use a physics-based model guided by fiducials landmarks identified by optical imaging thanks to their fluorescent characteristic.

The overall computational flow of our method involves two main steps: (1) Motion estimation of the kidney from laparoscopic images and (2) Computation of its deformations.

The mechanical representation allows to recover a physically coherent elastic deformations of the object and to estimate in-depth motion of the tumors.

In our case, tetraheadra-based FEM model implemented in the framework SOFA is used while mechanical parameters (Young modulus, Poisson ratio) are taken from the literature $[17,18]$.

The organ surface was manually segmented from the CT images using the Snake algorithm available in the opensource software (itk-SNAP, available online at: http:// www.itksnap.org). In order to build the mechanical model, a volume was automatically generated using the 3D Delaunay triangulation algorithm which is available in the CGal library (The Computational Geometry Algorithms Library; available at http://www.cgal.org/).

The spatial information of the fiducial was obtained from the laparoscopic image, and the re-localization of the fiducial was made automatically between the laparoscopic image and the virtual model. This re-localization is performed using a Perspective-n-Point algorithm (PnP).

The aim of the PnP algorithm is to determine the position and orientation of a camera given its intrinsic parameters and a set of correspondences between 3D points and their $2 \mathrm{D}$ projections. In our case, the $3 \mathrm{D}$ points are coupled with the biomechanical model while the $2 \mathrm{D}$ projections represent the fiducials.

Then sequential computation of the FEM deformation and the PnP re-localization are performed simultaneously. 
Once the computation has been performed, the surfaces are projected onto the laparoscopic images thanks to the camera's intrinsic parameters to obtain the final render that will be displayed to the surgeon.

\section{Results}

\section{Ex vivo experiments}

All the six fiducials in each kidney were successfully placed with less than $2 \mathrm{~mm}$ of exposed tip. A fluorescent signal allowed easy and clear identification of the fiducials in spite of the small exposed area of the fiducials, compared with naked-eye observation (Fig. 2). With dimmed background light, the shape of the kidney could be visualized together with the fluorescent signal of the fiducials (Fig. 2C, F).

Flexible virtual models were successfully generated from the CT scan of the kidney (Fig. 3). The fiducials generated some artifacts; however, all the artifacts could be adjusted by digital modification. The mean long and short axis lengths of the kidneys were $100.2 \pm 9.8$ and $50.0 \pm 3.0 \mathrm{~mm}$, respectively. The number of vertices made from segmentation of the surface ranged from 10,968 to 13,744 . The volume model was automatically generated with tetrahedron meshes. The number of the tetrahedral meshes ranged from 3072 to 4096 . The final flexible virtual model was established by combining the surface model and the volume model, as well as the location of the fiducials and the pseudo-tumors.

When the kidney was deformed, changed locations of fiducials were identified by the near-infrared camera (Fig. 4A, B). The new locations of the fiducials were automatically found with the fiducials of the virtual model. The biomechanical model was deformed automatically according to the re-localized position of the fiducials, resulting in the change of location of the pseudo-tumors as well (Fig. 4C, D). The biomechanical virtual models could be superimposed on the image of the kidney indicating the location of the pseudo-tumors inside the parenchyma (Fig. 4E, F). The error between the locations of the pseudotumors in the ground truth data obtained from CT scan and the predicted location of virtual biomechanical model in deformed shape was $0.76 \pm 0.23 \mathrm{~mm}$ for the pseudo-tumors placed in the parenchyma on the same side where the fiducials were placed, and $0.92 \pm 0.55 \mathrm{~mm}$ for the pseudotumors placed on the side opposite the fiducials (Fig. 4D, $\mathrm{G).}$

\section{In vivo experiments}

All six fiducials were successfully placed on the surface of the kidney (Fig. 5) and could be clearly identified by the near-infrared fluorescence camera system in spite of their small caliber and of their position. In fact, some fiducials
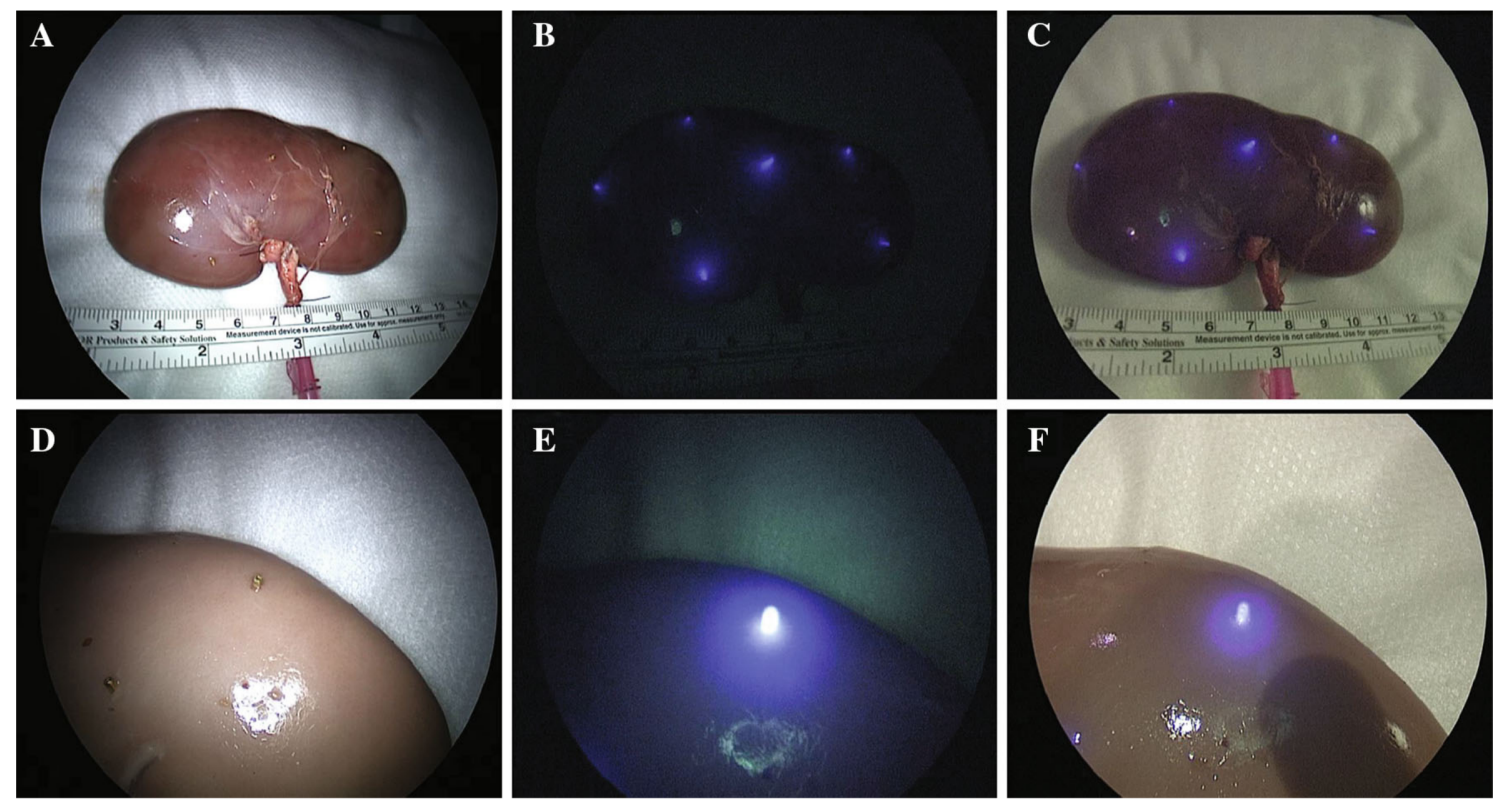

Fig. 2 Fiducial placement on the surface of the ex vivo kidneys; A, D white light mode; B, E near-infrared mode without background light; $\mathbf{C}$, F near-infrared mode with dimmed background light 

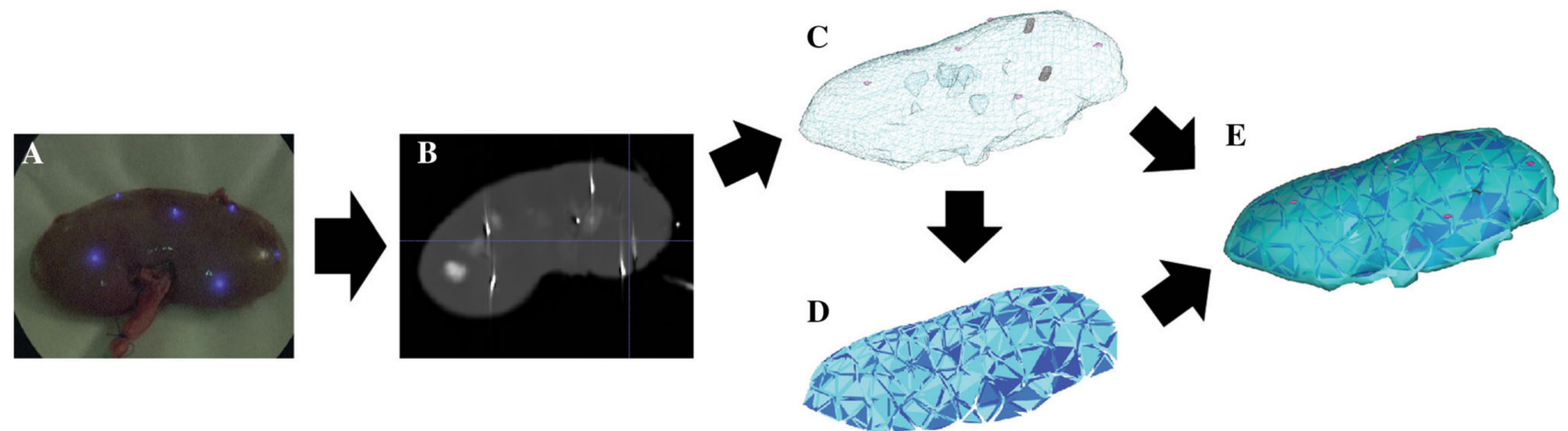

Fig. 3 Generation of virtual models; A ex vivo kidney with fluorescent surface fiducials; B CT scan of the kidney; C segmentation of the surface of the kidney; $\mathbf{D}$ volume model automatically generated

from the segmented surface; $\mathbf{E}$ final biomechanical model combining the surface mode, the volume model, fiducials, and the pseudo-tumors

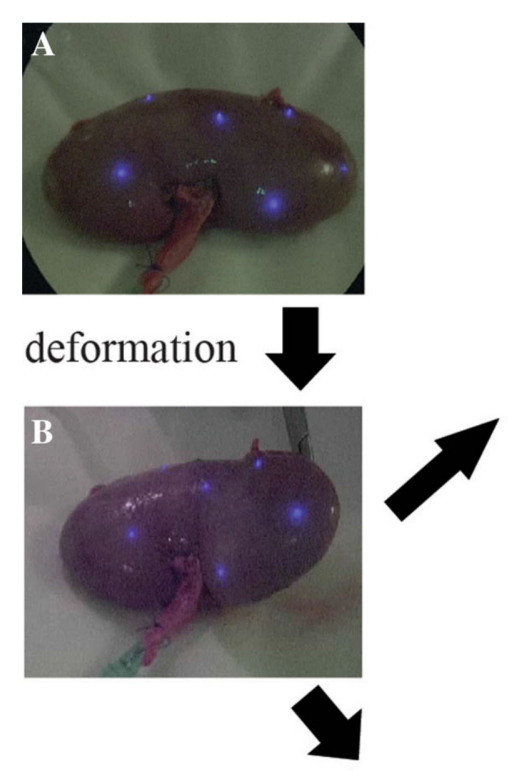

C

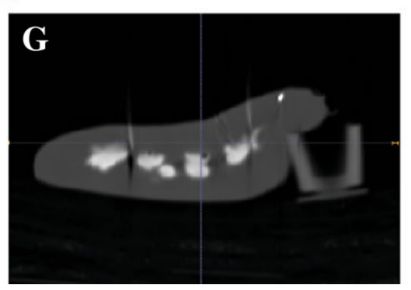

Fig. 4 Re-localization of the position of the fiducials and automatic deformation of the virtual model; A, B Deformation of the kidney in the laparoscopic view; C, D deformation of the virtual model following automatic re-localization of the position of the fiducials; $\mathbf{E}$,

were partially surrounded by hematoma (Fig. 5C, D), and some were relatively deeply placed with only a very small part of the fiducial exposed under Gerota's fascia (Fig. 5E, F). Most of the injection sites induced self-limiting subcapsular hematomas, but no active bleeding.

The virtual model was generated from the CT scan, and the model was overlaid on the laparoscopic image by relocalization of the place of surface fiducials (Fig. 6). Dice's coefficient comparing the boundary of the kidney between
F overlaid virtual models on the laparoscopic view indicating the location of the pseudo-tumors; $\mathbf{G}$ ground truth of deformed kidney obtained by CT scan; $\mathbf{H}$ overlay of the virtual model and the segmented surface of the ground truth in CT scan

the virtual model and the laparoscopic image was 0.95 , which means $95 \%$ of similarity (Fig. 6C).

\section{Discussion}

Augmented reality is a promising technology for tailored resections of solid organ tumors, enabling to see by transparency and providing enhanced guidance for 

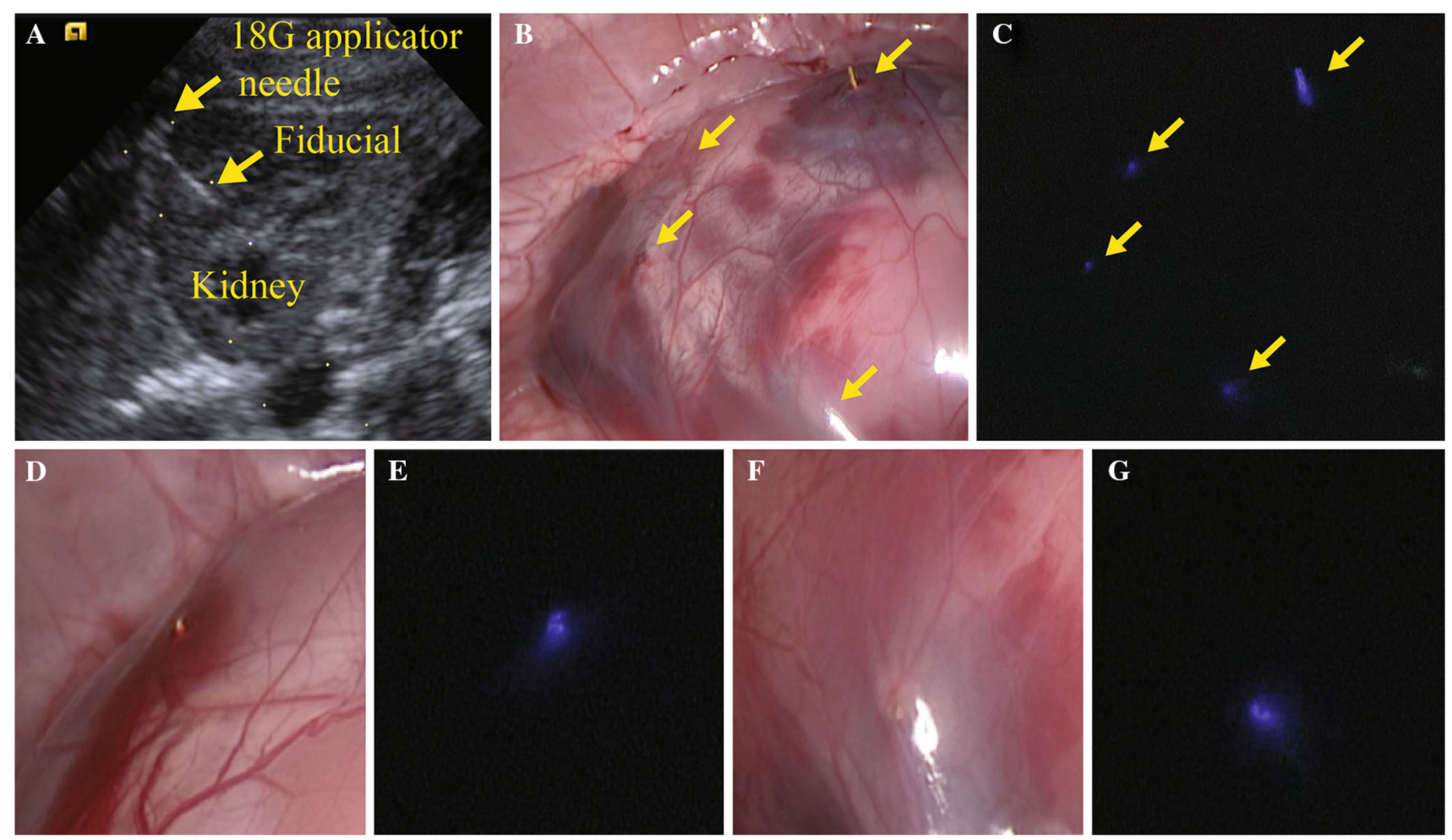

Fig. 5 Fiducial placement on the surface of in vivo kidney. A Placement of the fiducial with a guidance of ultrasonography; $\mathbf{B}$, D, and $\mathbf{F}$ white light mode; C, E, and $\mathbf{G}$ near-infrared mode; D, E fiducial surrounded by hematoma; F, G fiducial covered by the Gerota's fascia
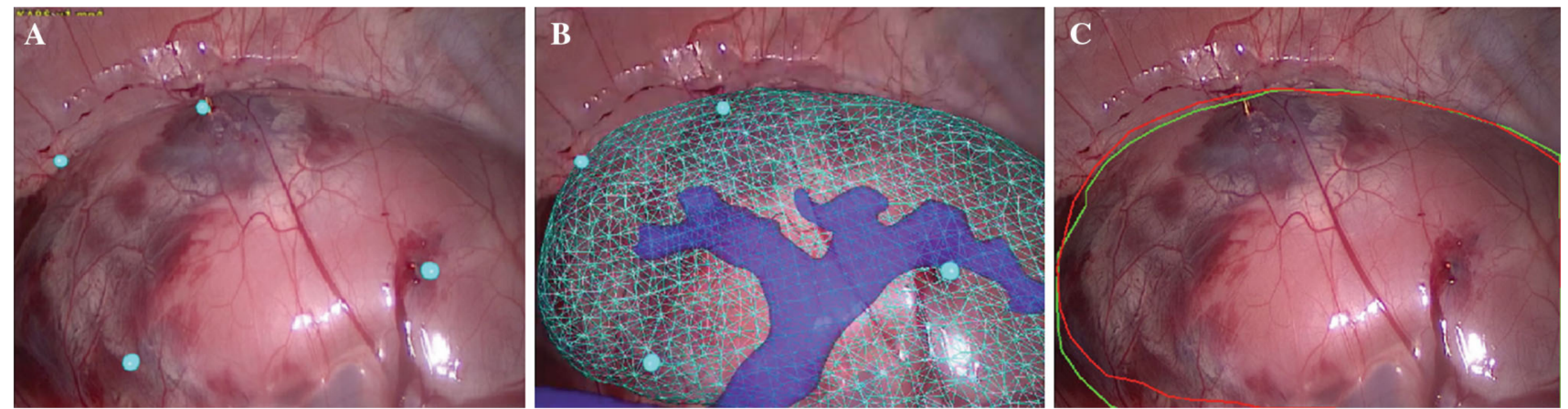

Fig. 6 Re-localization of the fiducials in in vivo kidney. A Identification of the fiducial from the laparoscopic image. B Overlay of the virtual model on the laparoscopic image by means of re-localization

precision surgery. However, AR is limited by the fact that the virtual model obtained from the imaging modality is "rigid" and does not follow the deformations of the real organ when it is manipulated during the surgical procedure.

Registration is the process of precisely fitting the 3D virtual model obtained from imaging onto the real patient's anatomy and is a critical step to provide correct and useful information to the surgeon. Registration is an area of actively ongoing research in computer-assisted imageguided surgery [19], and it can be rigid or non-rigid [20-22] and can be manual or automatic. Available registration methods involve some degree of human interaction of the fiducials $\mathbf{C}$ comparison of the boundary between the virtual model (red line) and the estimated border in the laparoscopic image (green line) (Color figure online)

in visualizing the preoperative 3D model directly on the operative monitor and manually resize and orientate the model according to some landmarks (e.g., bone structures such as the iliac crest or potentially some radio-opaque markers used during both the acquisition of the preoperative images and intraoperatively) [23-26]. Alternatively, landmark position is initially determined on the preoperative image and subsequently outlined during the procedure with a navigation pointer [27], and the model is then semiautomatically repositioned. Ieiri et al. [28] recently applied an optical tracking system for registration between volume image and skin markers to guide laparoscopic splenectomy 
in six children. The authors reported an acceptable registration accuracy of the system in the clinical setting and the ability of the navigation to provide real-time anatomical information, which could not otherwise have been visualized.

The main problems with automatic registration include organ deformation or displacement by surgical manipulation. The ability to perform a real-time intraoperative update of the 3D model of the targeted organ or at least estimate the deformation range with good approximation is the key point for fully automatic AR registration. The most straightforward approach is to directly acquire a novel 3D image of the interest zone during the procedure which could be updated at any time during surgery. This can be done using 3D ultrasound, as recently demonstrated by Nam et al. [29] for liver surgery applications, or by CT scan [30] or MRI. Shekhar et al. [30] proposed to use an intraoperative low-dose CT scan image to update the 3D liver model and refresh the AR view during surgical manipulation. The authors also used an optical tracking system to follow the endoscopic camera. Although interesting, a drawback of this approach that should be noted is related to the low-dose CT scan which provides less detailed images. The second inconvenient is the need for a CT scan during the whole procedure. Additionally, the update rate of the 3D virtual model should be swift enough to be of clinical utility during a surgical procedure.

To enable automatic registration of the 3D model after being deformed, we propose to use surface fiducials which can be identified both with CT scan and in the laparoscopic image as strong landmarks with high consistency and accuracy. Additionally, since fiducials are supposed to be placed around the tumor, most of them remain within the laparoscopic view and can be tracked throughout the whole surgical procedure, without the risk of being lost by zooming into the focal area around the tumor during fine dissection. In the present study, near-infrared fluorescent coating was found to be useful to enhance the visibility of the surface fiducials. Near-infrared wavelength $(700-900 \mathrm{~nm})$ is the range of light that is the least absorbed by human tissues, providing high signal-to-background ratio and deep penetration up to $5-10 \mathrm{~mm}$ of tissue [31]. In this experiment, the visibility of the fiducial in near-infrared mode was superior to that in white light mode, and the fiducials could be well visualized, even when they were partly surrounded by hematoma or covered by Gerota's fascia. As an additional advantage, the enhanced visibility could contribute to reduce the size of fiducials resulting in a reduced risk of injuries to organs.

Fluorescence is useful in automatic identification of fiducials in laparoscopic images, because in the near-infrared mode it is possible to selectively visualize the fluorescent fiducials. Unfortunately, the system used in the present experiments has a limitation, as operators had to toggle between the white light mode and the fluorescent mode. However, other commercially available laparoscopic systems (e.g., the Pinpoint from Novadaq, Mississauga, ON, Canada) can display both white light image and near-infrared image in overlay. Identification of the fiducials can be more robust and automatic using such systems enabling overlay. Alternatively, we could obtain the image similar to the superimposed image by adding another dimmed light source in our fluorescent camera system (Figure 2C, F).

Although re-localization is automatized, the rigid virtual model still has limitations in simulating the deformation of organs during operation. FEM model is a promising solution to simulate the elastic deformity of organs. In our last liver experiment, we showed that the FEM model can simulate the deformity during operation and be superimposed on the laparoscopic images simultaneously [11].

The prediction error of the virtual model concerning the deformation of the organ and the location of the pseudotumor was $<1 \mathrm{~mm}$. The virtual biomechanical kidney model was designed to follow dynamic simulation of flexibility and compressibility of the real organs. However, there are additional levels of structural complexity that were not computed, including the mechanical contribution of elements such as the renal capsule, cortex, medullar, and calyces, not to mention individual variations. By definition a model is not perfect. Nevertheless, the accuracy of this model was estimated to be acceptable when considering that the usual targeted tumor margins are around $0.5 \mathrm{~cm}$ in the clinical situation [32]. Because the segmentation of detailed structures in the organ requires additional and time-consuming manual work, it can be more cost-effective to apply an average value of the physical property to the whole organ.

We have selected the kidney for this proof of the concept for several reasons. First, the kidney is covered with fat tissue under Gerota's fascia, which prevents the use of organ boundaries as a landmark for AR registration, unless a significant portion of the kidney is exposed. Second, partial nephrectomy or nephron-sparing surgery has already been an established treatment strategy for $\mathrm{T} 1$ renal cell carcinoma, due to its comparable oncologic outcomes, preservation of renal function, and benefits on other causes of cause mortality compared with radical nephrectomy [33, 34]. Third, in spite of some debates, complete resection with a negative surgical margin is still regarded as a mainstay oncologic principle of the partial nephrectomy for renal cell carcinoma [35, 36]. Fourth, small tumors are frequently partially exposed in the capsule, covered by perinephric fat, or deep-seated with an endophytic 
growth pattern, so it is not always easy to localize the extent of the tumor and accurately delineate the margins [3, 37, 38]. Finally, the porcine kidney is technically easy to manipulate. Importantly, it is expected that the technique we propose can be applied to other solid organs, including, for example, the liver.

The fluorescent fiducials, outside the concept of AR, can be useful to improve the localization of superficial solid organs' tumors in the minimally invasive setting. In such cases, only 1-2 fiducials around the tumor may be sufficient to guide the surgeon. However, for a robust augmented reality, at least four fiducials are required to solve the PnP problem and calculate the position and the direction of the camera [39], which can represent an economic issue.

Moreover, in our experiment we had a self-limiting hematoma, despite the small size of the fiducial. The use of fiducials is considered relatively safe $[40,41]$, but a concern of delayed complications, such as chronic bleeding, should be further evaluated.

Therefore, our next development would be to find less invasive methods and materials for the surface fiducials. One of the solutions is to use a minimal number of fiducials at the beginning of the operation and to maximize the use of anatomical landmarks at the boundary or outside of the organ, and to add less invasive fiducials as needed during the operation. On the other hand, other researches to simulate the conditions during operations, such as an algorithm to simulate the virtual model during the cutting of the organ, [42] and the validation of the deformation of the virtual model under different intraabdominal pressure, are under investigation. Combining all of these, it is expected that real-time AR which can be applied to most intraoperative situations could be developed in the near future.

\section{Conclusions}

A flexible biomechanical 3D model with a fluorescent gold surface fiducial was feasible in a porcine model with acceptable accuracy and automatized robust tracking. The principle seems to be useful to provide accurate real-time visual information concerning the location and the margin of the small solid tumors during tailored operations. Further researches on the safety of fiducials and the development of less invasive surface marking methods are under investigation.

Acknowledgments Authors are grateful to Christopher Burel, professional in medical English proofreading, for their valuable help in revising the manuscript.

\section{Compliance with ethical standards}

Disclosures SH Kong, N Haouchine, R Soares, A Klymchenko, B Andreiuk, B Marques, G Shabat, T Piechaud, M Diana, S Cotin, and J Marescaux have no conflicts of interest or financial ties to disclose.

\section{References}

1. Kim SP, Thompson RH (2013) Kidney function after partial nephrectomy: current thinking. Curr Opin Urol 23:105-111

2. Tang YH, Wen TF, Chen X (2013) Anatomic versus non-anatomic liver resection for hepatocellular carcinoma: a systematic review. Hepato-gastroenterology 60:2019-2025

3. Hou W, Yan W, Ji Z (2015) Anatomic features involved in technical complexity of partial nephrectomy. Urology 85:1-7

4. Santambrogio R, Opocher E, Ceretti AP, Barabino M, Costa M, Leone S, Montorsi M (2007) Impact of intraoperative ultrasonography in laparoscopic liver surgery. Surg Endosc 21:181-188

5. Marescaux J, Diana M (2015) Next step in minimally invasive surgery: hybrid image-guided surgery. J Pediatr Surg 50:30-36

6. D’Agostino J, Diana M, Vix M, Soler L, Marescaux J (2012) Three-dimensional virtual neck exploration before parathyroidectomy. N Engl J Med 367:1072-1073

7. Marescaux J, Diana M (2015) Inventing the future of surgery. World J Surg 39:615-622

8. Marzano E, Piardi T, Soler L, Diana M, Mutter D, Marescaux J, Pessaux P (2013) Augmented reality-guided artery-first pancreatico-duodenectomy. J Gastrointest Surg 17:1980-1983

9. Pessaux P, Diana M, Soler L, Piardi T, Mutter D, Marescaux J (2014) Robotic duodenopancreatectomy assisted with augmented reality and real-time fluorescence guidance. Surg Endosc 28:2493-2498

10. Pessaux P, Diana M, Soler L, Piardi T, Mutter D, Marescaux J (2015) Towards cybernetic surgery: robotic and augmented reality-assisted liver segmentectomy. Langenbecks Arch Surg 400:381-385

11. Haouchine N, Cotin S, Peterlik I, Dequidt J, Lopez MS, Kerrien E, Berger MO (2015) Impact of soft tissue heterogeneity on augmented reality for liver surgery. IEEE Trans Vis Comput Graph 21:584-597

12. Collins BT, Erickson K, Reichner CA, Collins SP, Gagnon GJ, Dieterich S, McRae DA, Zhang Y, Yousefi S, Levy E, Chang T, Jamis-Dow C, Banovac F, Anderson ED (2007) Radical stereotactic radiosurgery with real-time tumor motion tracking in the treatment of small peripheral lung tumors. Radiat Oncol 2:39

13. Dieterich S, Gibbs IC (2011) The CyberKnife in clinical use: current roles, future expectations. Front Radiat Ther Oncol 43:181-194

14. Chavalitdhamrong D, DiMaio CJ, Siersema PD, Wagh MS (2015) Technical advances in endoscopic ultrasound-guided fiducial placement for the treatment of pancreatic cancer. Endosc Int Open 3:E373-E377

15. Kong SH, Noh YW, Suh YS, Park HS, Lee HJ, Kang KW, Kim HC, Lim YT, Yang HK (2015) Evaluation of the novel nearinfrared fluorescence tracers pullulan polymer nanogel and indocyanine green/gamma-glutamic acid complex for sentinel lymph node navigation surgery in large animal models. Gastric Cancer 18:55-64

16. Crum WR, Camara O, Hill DL (2006) Generalized overlap measures for evaluation and validation in medical image analysis. IEEE Trans Med Imaging 25:1451-1461 
17. Faure F, Duriez C, Delingette H, Allard J, Gilles B, Marchesseau S, Talbot H, Courtecuisse H, Bousquet G, Peterlik I (2012) Sofa: a multi-model framework for interactive physical simulation. Soft tissue biomechanical modeling for computer assisted surgery. Springer, New York, pp 283-321

18. Yamada H, Evans FG (1970) Strength of biological materials. Williams \& Wilkins, Philadelphia

19. Marescaux J, Diana M, Soler L (2013) Augmented reality and minimally invasive surgery. J Gastroenterol Hepatol Res 2:555-560

20. Pilet J, Lepetit V, Fua P (2008) Fast non-rigid surface detection, registration and realistic augmentation. Int $\mathbf{J}$ Comput Vis 76:109-122

21. Gay-Bellile V, Bartoli A, Sayd P (2010) Direct estimation of nonrigid registrations with image-based self-occlusion reasoning. IEEE Trans Pattern Anal 32:87-104

22. Haouchine N, Dequidt J, Berger MO, Cotin S (2015) Monocular $3 \mathrm{D}$ reconstruction and augmentation of elastic surfaces with selfocclusion handling. IEEE Trans Vis Comput Graph 21:1363-1376

23. Marescaux J, Rubino F, Arenas M, Mutter D, Soler L (2004) Augmented-reality-assisted laparoscopic adrenalectomy. JAMA J Am Med Assoc 292:2214-2215

24. Teber D, Guven S, Simpfendorfer T, Baumhauer M, Guven EO, Yencilek F, Gozen AS, Rassweiler J (2009) Augmented reality: a new tool to improve surgical accuracy during laparoscopic partial nephrectomy? Preliminary in vitro and in vivo results. Eur Urol 56:332-338

25. Chen Y, Li H, Wu D, Bi K, Liu C (2014) Surgical planning and manual image fusion based on 3D model facilitate laparoscopic partial nephrectomy for intrarenal tumors. World J Urol 32:1493-1499

26. Wang D, Zhang B, Yuan X, Zhang X, Liu C (2015) Preoperative planning and real-time assisted navigation by three-dimensional individual digital model in partial nephrectomy with three-dimensional laparoscopic system. Int J Comput Assist Radiol Surg 10:1461-1468

27. Marvik R, Lango T, Tangen GA, Andersen JO, Kaspersen JH, Ystgaard B, Sjolie E, Fougner R, Fjosne HE, Nagelhus Hernes TA (2004) Laparoscopic navigation pointer for three-dimensional image-guided surgery. Surg Endosc 18:1242-1248

28. Ieiri S, Uemura M, Konishi K, Souzaki R, Nagao Y, Tsutsumi N, Akahoshi T, Ohuchida K, Ohdaira T, Tomikawa M, Tanoue K, Hashizume M, Taguchi T (2012) Augmented reality navigation system for laparoscopic splenectomy in children based on preoperative CT image using optical tracking device. Pediatr Surg Int 28:341-346

29. Nam WH, Kang DG, Lee D, Lee JY, Ra JB (2012) Automatic registration between $3 \mathrm{D}$ intra-operative ultrasound and pre-operative CT images of the liver based on robust edge matching. Phys Med Biol 57:69-91

30. Shekhar R, Dandekar O, Bhat V, Philip M, Lei P, Godinez C, Sutton E, George I, Kavic S, Mezrich R, Park A (2010) Live augmented reality: a new visualization method for laparoscopic surgery using continuous volumetric computed tomography. Surg Endosc 24:1976-1985

31. Noh YW, Kong SH, Choi DY, Park HS, Yang HK, Lee HJ, Kim HC, Kang KW, Sung MH, Lim YT (2012) Near-infrared emitting polymer nanogels for efficient sentinel lymph node mapping. ACS Nano 6:7820-7831

32. Sutherland SE, Resnick MI, Maclennan GT, Goldman HB (2002) Does the size of the surgical margin in partial nephrectomy for renal cell cancer really matter? J Urol 167:61-64

33. Kim SP, Thompson RH, Boorjian SA, Weight CJ, Han LC, Murad MH, Shippee ND, Erwin PJ, Costello BA, Chow GK, Leibovich BC (2012) Comparative effectiveness for survival and renal function of partial and radical nephrectomy for localized renal tumors: a systematic review and meta-analysis. J Urol 188:51-57

34. Sun M, Trinh QD, Bianchi M, Hansen J, Hanna N, Abdollah F, Shariat SF, Briganti A, Montorsi F, Perrotte P, Karakiewicz PI (2012) A non-cancer-related survival benefit is associated with partial nephrectomy. Eur Urol 61:725-731

35. Lam JS, Bergman J, Breda A, Schulam PG (2008) Importance of surgical margins in the management of renal cell carcinoma. Nat Clin Pract Urol 5:308-317

36. Marszalek M, Carini M, Chlosta P, Jeschke K, Kirkali Z, Knuchel R, Madersbacher S, Patard JJ, Van Poppel H (2012) Positive surgical margins after nephron-sparing surgery. Eur Urol 61:757-763

37. Di Pierro GB, Tartaglia N, Aresu L, Polara A, Cielo A, Cristini C, Grande P, Gentile V, Grosso G (2014) Laparoscopic partial nephrectomy for endophytic hilar tumors: feasibility and outcomes. Eur J Surg Oncol 40:769-774

38. Autorino R, Khalifeh A, Laydner H, Samarasekera D, Rizkala E, Eyraud R, Stein RJ, Haber GP, Kaouk JH (2014) Robot-assisted partial nephrectomy (RAPN) for completely endophytic renal masses: a single institution experience. BJU Int 113:762-768

39. Moreno-Noguer F, Lepetit V, Fua P (2007) Accurate non-iterative $o(n)$ solution to the pnp problem. In: IEEE 11th international conference on computer vision. IEEE, pp 1-8

40. Kim JH, Hong SS, Kim JH, Park HJ, Chang YW, Chang AR, Kwon SB (2012) Safety and efficacy of ultrasound-guided fiducial marker implantation for CyberKnife radiation therapy. Korean J Radiol 13:307-313

41. Kothary N, Heit JJ, Louie JD, Kuo WT, Loo BW Jr, Koong A, Chang DT, Hovsepian D, Sze DY, Hofmann LV (2009) Safety and efficacy of percutaneous fiducial marker implantation for image-guided radiation therapy. J Vasc Interv Radiol JVIR 20:235-239

42. Paulus CJ, Haouchine N, Cazier D, Cotin S (2015) Surgical augmented reality with topological changes. International conference on medical image computing and computer-assisted intervention. Springer, New York, pp 413-420 